\title{
Prenatal Testing for Adult-Onset Conditions: the Position of the National Society of Genetic Counselors
}

\author{
Laura Hercher ${ }^{1}$ - Wendy R. Uhlmann ${ }^{2}$ Erin P. Hoffman ${ }^{3}$ - Shanna Gustafson ${ }^{4}$. \\ Kelly M. Chen ${ }^{5}$ the Public Policy Committee of NSGC
}

Received: 25 May 2016 / Accepted: 16 June 2016/Published online: 1 July 2016

(C) National Society of Genetic Counselors, Inc. 2016

\begin{abstract}
Advances in genetic testing and the availability of such testing in pregnancy allows prospective parents to test their future child for adult-onset conditions. This ability raises several complex ethical issues. Prospective parents have reproductive rights to obtain information about their fetus. This information may or may not alter pregnancy management. These rights can be in conflict with the rights of the future individual, who will be denied the right to elect or decline testing. This paper highlights the complexity of these issues, details discussions that went into the National Society of Genetic Counselors (NSGC) Public Policy Task Force's development of the Prenatal testing for Adult-Onset Conditions position statement adopted in November 2014, and cites relevant literature on this topic through December 2015. Issues addressed include parental rights and autonomy, rights of the future child, the right not to know, possible adverse effects on childhood and the need for genetic counseling. This paper will serve as a reference to genetic counselors and healthcare professionals when faced with this situation in clinical practice.
\end{abstract}

Kelly M. Chen

km_chen@yahoo.com

the Public Policy Committee of NSGC

1 Joan H. Marks Graduate Program in Human Genetics, Sarah Lawrence College, NY, USA

2 Department of Internal Medicine and Department of Human Genetics, University of Michigan, Ann Arbor, MI 48109, USA

3 Department of Oncology, Littleton Adventist Hospital, Littleton, CO, USA

4 Informed DNA, Saint Petersburg, FL, USA

5 Personalis Inc, 1330 O’Brien Drive, Menlo Park, CA 94025, USA
Keywords Prenatal testing · Adult-onset conditions · Genetic counseling $\cdot$ Reproductive rights $\cdot$ Ethical issues $\cdot$ The National Society of genetic counselors $\cdot$ Position statement

\section{Introduction}

The National Society of Genetic Counselors (NSGC) releases position statements that are intended to convey to the public the Society's specific views and opinions on issues of relevance to the practice of genetic counseling. The NSGC Public Policy Committee (PPC) leads the creation of new statements, or revision of existing statements, based on emerging data or issues. This paper highlights the background information that informed the Task Force members' discussions and shaped the statement on prenatal testing for adult-onset conditions put forward to the NSGC membership and Board of Directors for comments and approval, and adopted on November 3, 2014.

NSGC's position statement on prenatal testing for adult-onset conditions is as follows: "The National Society of Genetic Counselors (NSGC) encourages deferring prenatal genetic testing for adult-onset conditions if pregnancy management will not be affected. Prospective parents have the right to make fully informed and autonomous decisions about reproductive options and pregnancy management. However, prenatal testing for adult-onset conditions denies the future child the opportunity to make this decision for him/herself as an adult. NSGC strongly recommends that prospective parents meet with a certified genetic counselor or other healthcare specialist with genetic counseling expertise for decision-making about prenatal testing. Adopted by NSGC on 11/3/14" (NSGC Position Statement Prenatal Testing for Adult-Onset Conditions 2014). 


\section{Background}

As a result of advances in genetics, more genes are being identified for adult-onset conditions and there is increased availability of genetic testing. For some adult-onset genetic conditions (e.g. hereditary breast cancer, hereditary non-polyposis colon cancer), genetic testing is offered so that disease surveillance and, in some cases, prevention, can be initiated in adulthood prior to the onset of symptoms. For other conditions (e.g. Huntington disease, early-onset Alzheimer's disease), presymptomatic interventions are not available and testing may be done to learn genetic status and inform life decisions.

Prenatal use of genetic testing raises its own set of issues. The variety of genetic tests, both screening and diagnostic, available to the prospective parent continues to expand and can provide results as early as 9 weeks gestation (ACOG 2015). Prenatal genetic testing, in general, is now less expensive, quicker and safer than in the past. Results can take days rather than weeks, allowing for timely reproductive decisionmaking. The increased availability of prenatal testing options has raised both awareness among patients and ethical concerns that challenge healthcare professionals. (Van Lith et al. 2015; Wilson et al. 2015).

The National Society of Genetic Counselors (NSGC) and other professional organizations have discouraged testing minors for adult-onset conditions, and they recommend deferring testing until the individual reaches the age of majority unless there is a compelling reason to do otherwise (ASHG/ACMG 1995; Botkin et al. 2015; NSGC Position Statement Genetic Testing of Minors for Adult-Onset Conditions 2012; Ross et al. 2013). Prenatal testing for adult-onset conditions may allow tests considered inappropriate for minors to be performed in utero. If the pregnancy continues, this means that a minor will have been tested. However, denying the prospective parent the option of prenatal testing would create limitations in contradiction to the NSGC's position on reproductive freedom, which supports the rights of individuals and couples to make reproductive choices.

Negotiating this conflict between two principles with strong support in the genetic counseling community was the main concern of the Task Force as we prepared the NSGC position statement on prenatal testing for adult-onset conditions. As a Task Force, we felt strongly that a paper detailing the deliberations and research that informed our brief statement would be valuable, and serve to highlight the complicated ethical and practical issues that confront genetic counselors and other healthcare providers working in prenatal settings and other settings where patients are considering prenatal testing for adult-onset conditions.

The NSGC Public Policy Committee identified genetic counselors with experience and expertise in prenatal genetics, adult genetics/predictive testing, and ethics. The appointed Task Force researched other professional organizations' statements and conducted a literature review. Topics researched included prenatal, presymptomatic and childhood testing for adult-onset genetic conditions and pre-implantation genetic testing. Early in the discussions, the Task Force decided that the scope of the statement would focus on testing of an established pregnancy and not pre-implantation genetic diagnosis (PGD). The Task Force felt that the area of PGD involves a very different set of factors, players, and policies than prenatal testing.

From the literature search and conference call discussions, the Task Force delineated points to include in a position statement, which are discussed below. The Task Force developed the statement, submitted for a 2- week open member review, and collected and considered comments. The revised statement was sent to the NSGC Board of Directors for review and was adopted in November 2014.

In developing the position statement on prenatal testing for adult-onset conditions, the Task Force aimed to be consistent with other NSGC position statements especially the statements on Reproductive Freedom and genetic testing of Minors for Adult-Onset Conditions. NSGC's position statement on Reproductive Freedom supports the right of all individuals and couples to make reproductive choices, including the use of "information from genetic counseling and/or testing to decide whether to pursue a pregnancy,.... to prepare for the birth and future needs of their offspring, to make an adoption plan, or to end a pregnancy" (NSGC Position Statement: Reproductive Freedom 2010). The emphasis on patient autonomy and informed decisions is further supported by the NSGC Code of Ethics (NSGC Code of Ethics 1992). The NSGC Code of Ethics states that "The counselor-client relationship is based on values of care and respect for the client's autonomy, individuality, welfare and freedom" and that genetic counselors strive to "enable their clients to make informed decisions, free of coercion, by providing or illuminating the necessary facts, and clarifying the alternatives and anticipated consequences."

NSGC's position statement on Genetic Testing of Minors for Adult-Onset Conditions, adopted in 2012, "encourages deferring predictive genetic testing of minors for adult-onset conditions whenever possible" to "allow individuals to choose for themselves as adults, taking into account their own circumstances, preferences and beliefs." Guidelines from professional organizations, including the American College of Medical Genetics and Genomics, the American College of Pediatrics and the American Society of Human Genetics, (ASHG/ ACMG 1995; Botkin et al. 2015; Ross et al. 2013) all support deferring genetic testing of minors except in those circumstances when the genotypic information has some immediate benefit such as treatment or intervention in childhood or where testing would "alleviate substantial psychosocial distress" (Botkin et al. 2015).

While we found position statements on the topic of testing of minors, there was little that specifically addressed testing of a fetus in pregnancy for adult-onset conditions. A 2008 statement from the American College of Obstetricians and 
Gynecologists on "Ethical Issues in Genetic Testing," (ACOG 2008) reaffirmed in 2014, served mainly to frame the issue: "In pregnancies likely to be carried to term, consideration should be given to whether, as in the case of testing children, the decision to test should be reserved for the child to make upon reaching adulthood. However, consideration also should be given to personal preference, that is, the interests individuals may have in terminating a pregnancy that may result in a life (such as life that will be affected by Huntington chorea) that they feel morally obliged or prefer not to bring into the world."

The predictive genetic testing guidelines for Huntington disease (HD), which have been adapted for testing for other autosomal dominant neurogenetic conditions (e.g. early-onset Alzheimer's disease, frontotemporal dementias), clearly state that prenatal genetic testing should not be done if the pregnancy will be continued regardless of the HD results on the grounds that continuing a pregnancy after obtaining a positive result for HD would be akin to testing a minor and would deprive the future child their autonomy in deciding whether or not to learn this information (MacLeod et al. 2013).

\section{Ethical Considerations}

Prenatal and childhood testing for adult-onset conditions raises several ethical considerations. There are issues around autonomy including whose autonomy takes precedence - the parent(s) or the future child. The parent has the right to test the pregnancy and to decide whether or not to continue the pregnancy based on this information. However, if the parent is going to continue the pregnancy regardless of the results, then there is the issue that the genetic status of a minor will be known. Testing is offered to give prospective parents the ability to obtain information about the genetic status of their fetus and provide the option of termination, but cannot be done with the expectation of termination.

\section{Reproductive Rights}

The Task Force's statement on prenatal testing for adult-onset conditions clearly supports the prospective parents' right to know about the genetic status of a fetus, which is consistent with NSGC's position statement on reproductive freedom, but specifically encourages deferral if pregnancy management will not be affected.

The Task Force had lengthy discussions on whether or not to consider the prospective parents' motivation and intentions as a part of the decision regarding when to offer or decline testing. Should parents have the right to test regardless of their intended use of the information? Is it ethical for a healthcare provider or laboratory to determine whether prospective parents' reason for prenatal testing is sufficient or acceptable to proceed with testing? While prospective parents' inclinations are obviously an appropriate and important subject to address in counseling, in the opinion of the Task Force, parents could not be offered or denied testing based on a hypothetical discussion of their intentions. Answering hypothetical questions is not a genuine proxy for being confronted with real results. Prospective parents cannot be expected to focus the same way on the decision when they are asked about a risk and not a definite reality. Additionally, parents who say they either will or will not terminate a pregnancy can change their minds.

Genuine reproductive choice is contingent on the access to information. Although prospective parents are encouraged to carefully consider their intentions in proceeding with prenatal genetic testing, in the end, they should be provided with all the information they need, including test results if that is their choice, before making a decision about whether or not to continue or terminate a pregnancy. Expecting fully informed decisions will be made in advance is not a reasonable expectation.

\section{Rights of The Future Child}

Controversy exists with prenatal testing for an adult-onset condition because the results generally will not be used to affect the child's medical care in childhood. Therefore, it seems likely that the parent is making the decision to test a pregnancy out of a desire to know and not to benefit the child's care. The child's ability to exercise his/her future autonomy to decide whether to learn his/her genetic status and right to an open future is taken away if the parent determines the status prenatally.

Considering beneficence/non-maleficence, there are potential harms that could result from the child having his/her genetic status known including having this knowledge forced upon him/her, being raised as a "vulnerable child" and potential for stigmatization and insurance discrimination (ASHG/ ACMG 1995; Green and Solnit 1964). In the absence of using test results to affect pregnancy management or care during childhood, the future child's best interest needs to be a significant consideration in testing decisions.

\section{The Right not to Know}

The idea that we should decline to test minors based on the need to preserve their autonomy is closely tied to broader arguments in support of 'the right not to know.' The "right not to know" is frequently discussed with regard to genetic testing, and reflects a belief that rational individuals might decline to receive the type of predictive information that can be obtained from genetic testing, either because they would prefer not to know, the results may not impact their medical or life decisions, or because they are concerned about discrimination or stigma (Borry et al. 2014; Knoppers 2014; Takala 1999). Patients can 
decline medical care or diagnostic testing that could directly impact their physical health. Similarly, patients can decline predictive genetic testing even though there is a clear genetic risk and established screening. For example, patients at $50 \%$ risk to inherit an autosomal dominant gene for breast or colon cancer do decline genetic testing and the opportunity to perform preventative mammograms and colonoscopy (Leennen et al. 2016; National Comprehensive Cancer Network et al. 2015). In summary, patients often make testing and management decisions and as a consequence decline screening or treatment options, but ultimately this is their decision to make.

Arguments surrounding the "right not to know" stem from early discussions focused largely on highly penetrant and devastating neurological conditions such as Huntington disease (HD) and early-onset Alzheimer disease (AD). Uptake of the test for HD when it became available did not match the hypothetical demand, suggesting that many at-risk individuals who had expressed an interest in testing decided against it when presented with the actual option. (Bernhardt et al. 2009; Creighton et al. 2003; Tassicker et al. 2009).

The "right not to know" is considered grounds on which an individual can decline predictive genetic testing for him/herself and can be applied to the recommendation to refuse parental requests for genetic testing of their minor children for adultonset conditions. Our reluctance to permit parental consent in these cases suggests a strong proclivity to defer testing, and a high bar for what constitutes a necessary test. This approach similarly extends to prenatal testing for adult-onset conditions when pregnancy management will not change with the results.

\section{Adverse Effects on Childhood}

The Task Force found evidence in the literature of concern about the possible negative consequences of early access to genetic information on later onset conditions. These potential ill effects - on self-esteem, on relationships between parent and child, in terms of stigma or discrimination - are the subject of much discussion, but few studies exist (Michie et al. 2001; Pelias 2006). The idea of a "vulnerable child syndrome" was first introduced by Green and Solnit in 1965, who suggested that parents may become unduly overprotective or restrictive of a child deemed "at-risk." A 1995 paper published jointly by the American Society for Human Genetics (ASHG) and the American College of Medical Genetics has a detailed list of potential harms: a child may be over-indulged or treated as a scapegoat, results may alter their self-image, they may have survivor guilt or over-identify with affected family members, their privacy may be compromised. In 2015, ASHG published a Points to Consider document (Botkin et al. 2015) which focused on ethical, social and legal issues in the genetic testing of children associated with specific technology utilized, such as genome-scale sequencing, chromosome microarray, direct-to- consumer testing, pharmacogenomic testing, and newborn screening. The paper acknowledged that only a modest volume of clinical research has been conducted regarding the impact of predictive testing in high-risk families, but that this limited research has not found evidence of significant psychosocial harms in children.

Well-structured, systematic studies have yet to be performed to examine the psychosocial outcomes for children who undergo genetic testing. Wade et al. (2010) performed a review of literature spanning 30 years to identify studies that assessed the impact of communicating carrier (e.g. cystic fibrosis, TaySachs disease) or presymptomatic (e.g. familial adenomatous polyposis, hypertrophic cardiomyopathy) genetic test results to children. Although there was little quantitative evidence of negative impact of genetic test results on a child's psychosocial wellbeing, it was recognized that inconsistencies with methodology and psychopathology measures and small samples made it difficult for the authors to make any firm conclusions.

In the absence of outcomes data, the theoretical concerns regarding the impact of genetic testing of minors for adult onset conditions can be applied to prenatal disclosure of gene status, and ethical issues such as the right not to know or the right of a child to an open future must be seriously considered. In encouraging deferral of testing in situations where it will not alter pregnancy management, we emphasize the importance of protecting the best interests of the future child. However, we recognize the reproductive rights of prospective parents as paramount when conflicts cannot be resolved. As a consequence of protecting the parents' reproductive rights, we accept that at times children will be born having already had genetic tests which are widely considered inappropriate for minors. To keep those circumstances to a minimum, we recommend that prospective parents are counseled by genetics professionals and consider the use of other consultations as necessary, including a hospital ethics board and the NSGC Ethics Advisory Group.

\section{Other Considerations}

Potential insurance ramifications are a concern when testing for adult-onset conditions prenatally. Although health insurance is currently a protected entity under federal law (The Genetic Information Nondiscrimination Act of 2008), longterm care insurance, long-term disability and life insurance are not covered in this legislation. There is great variation in state law (National Human Genome Research Institute Genome Statute and Legislation Database n.d.), and not all of these laws would prevent insurers from denying a policy based on a pre-existing genetic test result. For individuals facing the possibility of a neurodegenerative or other severe disease, maintaining access to long-term care and long-term disability insurance is a significant consideration. Theoretically, individuals may be denied long-term care or life 
insurance based on family history. However, a positive genetic test may increase that likelihood, and the individual may be seen as pre-symptomatic, rather than at-risk. If genetic testing occurs prenatally, it removes the option for the future individual to procure appropriate insurance protection before seeking a risk assessment or genetic diagnosis. This risk of insurance discrimination is further complicated by the parental decision to disclose or not disclose this information to the future individual, who will be unaware of his/her genetic testing status.

Another issue raised in our deliberations, and in the response from the NSGC membership, was whether or not to consider characteristics of the condition as a part of the decision about whether or not to proceed with prenatal testing. The Task Force also discussed the ever-growing menu of genetic testing both in technique and scope of conditions offered.

Testing falls into two main categories: targeted and nontargeted. For the purposes of our discussion we defined targeted as a test for a single gene associated with a known disorder suspected in the patient, and non-targeted as any multi-gene test including panels (including targeted panels), array genomic hybridization, or whole exome sequencing. During pregnancy, targeted testing for a given gene or condition can be performed if there is a positive family history, abnormal ultrasound and/or other prenatal findings suggestive of a specific condition. For targeted testing, pretest counseling can reasonably include specific considerations including severity, age of onset, degree of penetrance and the availability of treatment. Prospective parents may have personal or familial experience with the condition under consideration, which may influence their perception of risk and drive their testing decisions. Recommendations for pretest counseling often presume that these aspects of the disease can and should be discussed with prospective parents before testing.

Non-targeted testing may be performed when a genetic condition is suspected but has not been identified, when a diagnosis has been made but the genetic etiology is unknown, or as a screen in the absence of any family history or medical finding. In this sense non-targeted testing changes the nature of pre-test counseling and may challenge current standards. In consideration of this issue we did not think it was wise or practical to tether the position statement on prenatal testing for adult-onset conditions to any specific set of circumstances or conditions. We acknowledge that as the use of prenatal testing changes, it may be necessary to re-visit this issue and provide revised guidelines regarding appropriate use of testing.

\section{Need for Pre-Test Genetic Counseling}

Given the complex issues raised by prenatal genetic testing for adult-onset conditions, we thought it was important to strongly recommend that prospective parents receive pre-and posttest genetic counseling. In addition, feedback from the NSGC membership stressed the need for counseling with a clinician with genetics expertise in order to help prospective parents understand these complex testing issues and the responsibilities associated with becoming custodians of their future child's genetic information. Genetic counseling should occur before testing is performed to ensure parents understand the practical and ethical considerations and provide informed consent for testing. It is equally important for parents to receive post-test counseling once results are available to ensure that they understand the implications and to explore pregnancy management options. As discussed above, parents may intend to terminate a pregnancy for a positive result, but could change their minds once results are tangible.

The Task Force carefully worded this recommendation for genetic counseling to emphasize the expertise needed. We did not want to suggest that the interest in counseling was tied only to genetic counselors and wanted to be inclusive in recognizing providers who have this expertise. Other NSGC position statements have similarly included the need for genetic counseling. Consistent with this recommendation, the HD predictive testing guidelines regarding prenatal testing specifically note the need for "careful pre-test counselling by an informed professional" and having this occur in a "specialized (prenatal or genetics) centre." (MacLeod et al. 2013).

While the position statement uses the wording "meet with" we recognize that genetic counseling services may not be locally available and such discussions may take place through genetic counseling services offered by teleconferencing, phone or other modalities.

\section{Future Directions}

Rapidly changing genetic technology will continue to influence the scope of prenatal genetic testing. The Task Force recognized these likely changes and discussed how to best address them in the guidelines for prenatal testing for adultonset conditions. Genetic testing will likely move away from targeted, single gene testing for a specific indication and towards non-targeted genomic testing for the general pregnant population and have the capability of identifying gene variants for adult-onset conditions. Whole exome sequencing (WES), commonly performed post-birth, is now being offered prenatally in specific cases (Carss et al. 2014, Hillman et al. 2015). Eventually, WES may shift to the general low-risk pregnant population if studies support the validity and accuracy of testing and costs decrease (Gregg et al. 2013). Non-invasive prenatal tests are rapidly increasing in popularity and have been shown in principle to be capable of providing a full fetal genotype in the first trimester (Kitzman et al. 2012). Given the real possibility of continued advances, we decided not to focus on a specific testing methodology but rather write guidelines that could be applied to a broad range of testing. 
The increasing availability and marketing of genetic tests will likely increase both the healthcare providers' and the public's familiarity with genetics and the inherent uncertainty of predictive genetic information. Concerns such as stigma, genetic discrimination, vulnerable child syndrome and difficulties in obtaining insurance may or may not wane over time. Studies to examine patient outcomes of prenatal genetic testing for adultonset conditions will be helpful to assess the true uptake of testing and implications. The Task Force supports continued research to investigate patient outcomes of prenatal testing for adult-onset conditions and the ethical, legal and social impact.

\section{Conclusion}

We acknowledge the primacy to the prospective parents' right to make autonomous reproductive decisions, and the impossibility of requiring individuals to predict their own course of action in advance of being fully informed, which may, in some instances, include seeking and obtaining results of genetic tests. Genetic counseling is important to advise prospective parents of the possible consequences of prenatal genetic testing and why testing may not be in the best interests of their future child. However, any conflict between the right of prospective parents to obtain information and the right of the future child should generally be resolved in favor of the parents.

Acknowledgments We thank the NSGC Membership and Board of Directors for the valuable comments and feedback they provided in the formation and revisions of this position statement.

\section{Compliance with Ethical Standards}

Conflict of Interest Authors Laura Hercher, Wendy Uhlmann, Erin Hoffman, Shanna Gustafson, and Kelly Chen declare that they have no conflict of interest.

Animal Studies No animal studies were carried out by the authors for this article.

\section{References}

American College of Obstetricians and Gynecologists (2008). Ethical issues in genetic testing. ACOG Committee opinion no. 410 . Obstetrics and Gynecology, 111, 1495-1502.

American College of Obstetricians and Gynecologists (2015). Cell-free DNA screening for fetal aneuploidy. ACOG Committee opinion no. 640. Obstetrics and Gynecology, 126, e31-e37.

American Society of Human Genetics Board of Directors; American College of Medical Genetics; Board of Directors (1995). Points to consider: ethical, legal, and psychosocial implications of genetic testing in children and adolescents. American Journal of Human Genetics, 57, 1233-1241.
Bernhardt, C., Schwan, A., Kraus, P., Epplen, J., \& Kuntsmann, E. (2009). Decreasing uptake of predictive testing for Huntington's disease in a German Centre: 12 years' experience (1993-2004). European Journal of Human Genetics, 17, 295-300. doi:10.1038/ejhg.2008.164.

Borry, P., Shabani, M., \& Howard, H. (2014). Is there a right time to know? The right not to know and genetic testing in children. Journal of Law, Medicine and Ethics, 42, w19-w27. doi:10.1111/jlme.12115.

Botkin, J., Belmont, J., Berg, J., Berkman, B., Bombard, Y., Holm, I., Levy, H., et al. (2015). Points to consider: ethical, legal, and psychosocial implications of genetic testing in children and adolescents. American Journal of Human Genetics, 97, 6-21. doi:10.1016/j. ajhg.2015.07.013.

Carss, K., Hillman, S., Parthiban, V., McMullan, D., Mahrer, E., Kilby, M., \& Hurles, M. (2014). Exome sequencing improves genetic diagnosis of structural fetal abnormalities revealed by ultrasound Human Molecular Genetics, 23, 3269-3277. doi:10.1093/hmg/ddu038.

Creighton, S., Almqvist, E., MacGregor, D., Fernandez, B., Hogg, H., Beis, J., Welch, J., et al. (2003). Predictive, pre-natal and diagnostic genetic testing for Huntington's disease: the experience in Canada from 1987 to 2000. Clinical Genetics, 63, 462-475.

Green M, Solnit AJ (1964). Reaction to the threatened loss of a child: a vulnerable child syndrome. Pediatrics, 34, 53-66.

Gregg, A., Gross, S., Best, R., Monaghan, K., Bajaj, K., Skotko, B., Thompson, B., et al. (2013). ACMG statement on noninvasive prenatal screening for fetal aneuploidy Genetics in Medicine, 15, 395388. doi:10.1038/gim.2013.29.

Hillman, S., Williams, D., Carss, K., McMullan, D., Hurles, M., \& Kilby, M. D. (2015). Prenatal exome sequencing for fetuses with structural abnormalities: the next step. Ultrasound in Obstetrics \& Gynecology, 45, 4-9. doi:10.1002/uog.14653.

Kitzman, J., Snyder, M., Ventura, M., Lewis, A., Qiu, R., Simmons, L., Gammill, H., et al. (2012). Noninvasive whole-genome sequencing of a human fetus. Science Translational Medicine, 4, 137ra76. doi: 10.1126/scitranslmed.3004323.

Knoppers, B. M. (2014). From the right to know to the right not to know. Journal of Law, Medicine and Ethics, 42, 6-10. doi:10.1111 /jlme.12113.

Leennen, C. H., Heijer, M. D., van der Meer, C., Kuipers, E. J., van Leerdam, M. E., \& Wagner, A. (2016). Genetic testing for lynch syndrome: family communication and motivation. Familial Cancer, 15(1), 63-73. doi:10.1007/s10689-015-9842-8.

MacLeod, R., Tibben, A., Frontali, M., Evers-Kiebooms, G., Jones, A., Martinz-Descales, A., \& Editorial Committee and Working Group "Genetic testing Counselling" of the European Huntington Disease Network. (2013). Recommendations for the predictive genetic test in Huntington's disease. Clinical Genetics, 83, 221-231. doi:10.1111/j.1399-0004.2012.01900.

Michie, S., Bobrow, M., Marteau, T., \& on behalf of the FAP Collaborative Research Group. (2001). Predictive genetic testing in children and adults: a study of emotional impact. Journal of Medical Genetics, 38, 519-526. doi:10.1136/jmg.38.8.519.

National Comprehensive Cancer Network, NCCN Guidelines, Genetic/ Familial High Risk Assessment: Breast and Ovarian, 2.(2015). Retrieved November 1, 2015 from http://www.ncen.org/.

National Human Genome Research Institute Genome Statute and Legislation Database (n.d.). Retrieved from http://www.genome. gov/PolicyEthics/LegDatabase/pubsearch.cfm.

National Society of Genetic Counselors (1992). NSGC Code of Ethics. Retrieved November 1, 2015 from http://nsgc.org/p/cm/ld/fid=12.

National Society of Genetic Counselors (2010). NSGC Position Statement: Reproductive Freedom. Retrieved November 1, 2015 from $\mathrm{http}: / / \mathrm{nsgc}$.org/p/bl/et/blogaid=35.

National Society of Genetic Counselors (2012) NSGC Position Statement: Genetic Testing of Minors for Adult-Onset Conditions. Retrieved November 1, 2015 from http://nsgc.org/p/bl/et/blogaid=28. 
National Society of Genetic Counselors (2014). NSGC Position Statement: Prenatal Testing for Adult-Onset Conditions. Retrieved November 1, $2015 \mathrm{http}: / / \mathrm{nsgc}$.org/p/bl/et/blogaid=259.

Pelias, M. (2006). Genetic testing of children for adult-onset diseases: is testing in the child's best interests? Mt. Sinai Journal of Medicine, 73, 605-608.

Ross, L., Saal, H., David, K., Anderson, R., \& the American Academy of Pediatrics; American College of Medical Genetics and Genomics (2013). Technical report: ethical and policy issues in genetic testing and screening of children. Genetics in Medicine, 15, 234-245. doi:10.1038/gim.2012.176.

Takala, T. (1999). The right to genetic ignorance confirmed. Bioethics, 13, 288-293. doi:10.1111/1467-8519.00157.

Tassicker, R., Teltscher, B., Trembath, M., Collins, V., Sheffield, L., Chiu, E., Gurrin, L., \& Delatycki, M. (2009). Problems assessing uptake of Huntington disease predictive testing and a proposed solution.
European Journal of Human Genetics, 17, 66-70. doi:10.1038 lejhg.2008.

Van Lith, J., Faas, B., \& Bianchi, D. (2015). Current controversies in prenatal diagnosis 1: NIPT for chromosome abnormalities should be offered to women with low a priori risk. Prenatal Diagnosis, 35, 8-14. doi:10.1002/pd.4530.

Wade, C., Wilfond, B., \& McBride, C. (2010). Effects of genetic risk information on children's psychosocial wellbeing. Genetics in Medicine, 12, 317. doi:10.1097/GIM.0b013e3181de695c.

Wilson, R., Ledbetter, D., \& Pergament, E. (2015). Current controversies in prenatal diagnosis 3: the ethical and counseling implications of new genomic technologies: all pregnant women should be offered prenatal diagnostic genome-wide testing for prenatally identified fetal congenital anomalies. Prenatal Diagnosis, 35, 19-22. doi:10.1002/pd.4531. 